\title{
Cancellation ideals of a ring extension
}

\author{
S. Tchamna
}

\author{
Communicated by E. I. Zelmanov
}

\begin{abstract}
A BSTRACT. We study properties of cancellation ideals of ring extensions. Let $R \subseteq S$ be a ring extension. A nonzero $S$-regular ideal $I$ of $R$ is called a (quasi)-cancellation ideal of the ring extension $R \subseteq S$ if whenever $I B=I C$ for two $S$-regular (finitely generated) $R$-submodules $B$ and $C$ of $S$, then $B=C$. We show that a finitely generated ideal $I$ is a cancellation ideal of the ring extension $R \subseteq S$ if and only if $I$ is $S$-invertible.
\end{abstract}

\section{Introduction and background}

Throughout this article, we assume that all rings are commutative with identity. The notion of cancellation ideal for a ring has been studied in [1] and [2]. An ideal $I$ of a ring $R$ is called cancellation ideal if whenever $I B=I C$ for two ideals $B$ and $C$ of $R$, then $B=C$ [2]. A finitely generated ideal is a cancellation ideal if and only if for each maximal ideal $M$ of $R$, $I_{M}$ is a regular principal ideal of $R_{M}$ [1, Theorem 1]. D.D Anderson and D.F Anderson used the notion of cancellation ideal to characterize Prüfer domain. A ring $R$ is a Prüfer domain if and only if every finitely generated nonzero ideal of $R$ is a cancellation ideal[1, Theorem 6]. In this paper, we study the notion of cancellation ideal for ring extensions; which is a generalization of the notion of cancellation ideal for rings. Let $R \subseteq S$ be a ring extension, and let $A$ be an $R$-submodule of $S$. The $R$-submodule $A$ is said to be $S$-regular if $A S=S[5$, Definition 1, p. 84]. For two $R$-submodules $E, F$ of $S$, denote by $[E: F]$ the set of all $x \in S$ such that $x F \subseteq E$.

2020 MSC: 13A15, 13A18, 13B02.

Key words and phrases: ring extension, cancellation ideal, pullback diagram. 
An $R$-submodule $A$ of $S$ is said to be $S$-invertible, if there exists an $R$ submodule $B$ of $S$ such that $A B=R[5$, Definition 3, p 90]. In this case, we write $B=A^{-1}$, and $A^{-1}=[R: A]=\{x \in S: x A \subseteq R\}[5$, Remark 1.10, p. 90]. For the $R$-submodule $A$ of $S$, and for a multiplicative subset $\tau$ of $R$, we denote by $A_{[\tau]}$ the set of all $x \in S$ such that $t x \in A$ for some $t \in \tau$. If $\mathfrak{p}$ is a prime ideal of $R$, and $\tau=R \backslash \mathfrak{p}$, then $A_{[\mathfrak{p}]}$ denotes the set of all $x \in S$ such that $t x \in A$ for some $t \in \tau$. The set $A_{[\tau]}$ is called the saturation of $A$ by $\tau$. Properties of the saturation of a submodule are studied in [5, p. 18] and [6].

An $S$-regular ideal $I$ of $R$ is called (quasi)-cancellation ideal of the ring extension $R \subseteq S$ if whenever $I B=I C$ for two $S$-regular (finitely generated) $R$-submodules $B$ and $C$ of $S$, then $B=C$. In section 2, we study properties of (quasi)-cancellation ideals of ring extensions. In Proposition 2.4, we prove that a finitely generated $S$-regular ideal $I$ of $R$ is a cancellation ideal if and only it is a quasi-cancellation ideal. In Theorem 2.12, we show that for an $S$-regular finitely generated ideal $I$ of $R$, the followings are equivalent:

(1) $I$ is a cancellation ideal of the ring extension $R \subseteq S$.

(2) $I$ is an $S$-invertible ideal of $R$.

(3) $I R[X]$ is a cancellation ideal of the ring extension $R[X] \subseteq S[X]$.

Remark 1.1. Let $R \subseteq S$ be a ring extension, and let $A, B$ be two $R$ submodules of $S$. Then $A=B$ if and only if $A_{[\mathfrak{m}]}=B_{[\mathfrak{m}]}$ for each maximal ideal $\mathfrak{m}$ of $R$. In fact, if $A=B$, then it clear that $A_{[\mathfrak{m}]}=B_{[\mathfrak{m}]}$ for each maximal ideal $\mathfrak{m}$ of $R$. Conversely, if $A_{[\mathfrak{m}]}=B_{[\mathfrak{m}]}$ for each $\mathfrak{m} \in \mathcal{M}$, where $\mathcal{M}$ is the set of all maximal ideals of $R$, then by [5, Remark 5.5, p. 50], we have $A=\cap_{\mathfrak{m} \in \mathcal{M}} A_{[\mathfrak{m}]}=\cap_{\mathfrak{m} \in \mathcal{M}} B_{\mathfrak{m}}=B$.

Let $R \subseteq S$ and $L \subseteq T$ be two ring extensions, and consider the following commutative diagram

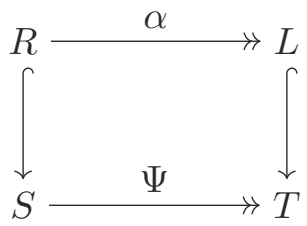

where $\operatorname{ker} \Psi$ is an ideal of $R, \Psi: S \longrightarrow T$ is surjective, the restriction $\alpha: R \longrightarrow L$ of $\Psi$ is also surjective and the vertical mappings are inclusions. When $\operatorname{ker} \Psi$ is a maximal ideal of $S$, the previous commutative diagram is called a pullback diagram a type $\square$. Pullback diagrams of type $\square$ are studied by S. Gabelli and E. Houston in [4]. 
Lemma 1.2. Consider the above pullback diagram of type $\square$. If $A, B$ are two $S$-regular ideals of $R$ such that $\Psi(A)=\Psi(B)$, then $A=B$.

Proof. Let $A, B$ be two $S$-regular ideals of $R$ such that $\Psi(A)=\Psi(B)$. By [7, Remark 1.1], we have ker $\Psi \subseteq A$ and ker $\Psi \subseteq B$. Let $a \in A$. Then there exists $b \in B$ such that $\Psi(a)=\Psi(b)$. Hence $a-b \in \operatorname{ker} \Psi \subseteq B$. Thus $a \in B$. This shows that $A \subseteq B$. With the same argument, $B \subseteq A$. Thus $A=B$.

\section{Cancellation ideals of ring extensions}

In this section, we define and study properties of cancellation ideals of ring extensions.

Definition 2.1. Let $R \subseteq S$ be a ring extension. A nonzero $S$-regular ideal $I$ of $R$ is called a (quasi)-cancellation ideal of the ring extension $R \subseteq S$ if whenever $I B=I C$ for two $S$-regular (finitely generated) $R$-submodules $B$ and $C$ of $S$, then $B=C$.

The following proposition studies cancellation ideals in pullback diagram of type $\square$. In this article, the Jacobson radical of a ring is denoted $\operatorname{Jac}(R)$.

Proposition 2.2. Suppose that the following diagram

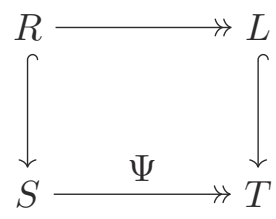

is a pullback diagram of type $\square$ such that $\operatorname{ker} \Psi \subseteq \operatorname{Jac}(R)$. Then an $S$ regular ideal $I$ of $R$ is a cancellation ideal of the extension $R \subseteq S$ if and only if $\Psi(I)$ is a cancellation ideal of the extension $L \subseteq T$.

Proof. Suppose that $I$ is a cancellation ideal of the extension $R \subseteq S$. Since $I S=S$, we have $\Psi(I) \Psi(S)=\Psi(S)$. It follows that $\Psi(I) T=T$. Hence $\Psi(I)$ is a $T$-regular ideal of $L$. Let $E$ and $F$ be two $T$-regular $L$-submodules of $T$ such that $\Psi(I) E=\Psi(I) F$. Let $B=\Psi^{-1}(E)$ and $C=\Psi^{-1}(F)$. Then by [7, Lemma 2.8(1)] $B$ and $C$ are two $S$-regular ideals of $R$. Furthermore, $E=\Psi(B)$ and $F=\Psi(C)$ since $\Psi$ is surjective. It follows from the equality $\Psi(I) E=\Psi(I) F$ that $\Psi(I) \Psi(B)=\Psi(I) \Psi(C)$. Hence $\Psi(I B)=\Psi(I C)$. Furthermore, $(I B) S=I S=S$ and $(I C) S=I S=S$. Therefore, by 
Lemma 1.2, we have $I B=I C$. Hence $B=C$ since $I$ is a cancellation ideal of the extension $R \subseteq S$. It follows that $E=\Psi(B)=\Psi(C)=F$. This shows that $\Psi(I)$ is a cancellation ideal of the extension $L \subseteq T$.

Conversely, suppose that $\Psi(I)$ is a cancellation ideal of the extension $L \subseteq T$. Let $B$ and $C$ be two $S$-regular $R$-submodules of $S$ such that $I B=$ $I C$. Then $\Psi(I) \Psi(B)=\Psi(I) \Psi(C)$. Since $B S=S$, we have $\Psi(B) T=T$. Hence $\Psi(B)$ is a $T$-regular ideal of $L$. With the same argument, $\Psi(C)$ is a $T$-regular ideal of $L$. It follows that $\Psi(B)=\Psi(C)$ since $\Psi(I)$ is a cancellation ideal of the extension $L \subseteq T$. Therefore, by Lemma 1.2, we have $B=C$. This shows that $I$ is a cancellation ideal of the extension $R \subseteq S$.

In the next proposition, we give a characterization of a cancellation ideal of a ring extension. This result is an analogue of [3, Proposition 2.1, p. $10]$ in the case of cancellation ideal of a ring.

Proposition 2.3. Let $R \subseteq S$ be a ring extension, and let I be an $S$-regular ideal of $R$. The following statements are equivalent.

(1) $I$ is a (quasi)-cancellation ideal of the ring extension $R \subseteq S$.

(2) $[I J: I]=J$ for any $S$-regular (finitely generated) $R$-submodule $J$ of $S$.

(3) If $I J \subseteq I K$ for two $S$-regular (finitely generated) $R$-submodules $J$ and $K$ of $S$, then $J \subseteq K$.

Proof. (1) $\Rightarrow(2)$ Suppose that $I$ is a cancellation ideal of the extension $R \subseteq S$, and let $J$ be an $S$-regular $R$-submodule of $S$. The containment $J \subseteq[I J: I]$ is always true. Let $x \in[I J: I]$. Then $x I \subseteq I J$. It follows that $(x, J) I \subseteq I J$, where $(x, J)$ is the $R$-submodule of $S$ generated by $x$ and $J$. Therefore, $(x, J) I=I J$ since the containment $I J \subseteq(x, J) I$ is always true. Furthermore, $(x, J)$ is an $S$-regular $R$-submodule of $S$ since $J \subseteq(x, J)$. It follows from the definition of a cancellation ideal that $(x, J)=J$. This shows that $x \in J$, and thus $[I J: I] \subseteq J$. Therefore $[I J: I]=J$.

$(2) \Rightarrow(3)$ Suppose that the statement (2) is true. Let $J$ and $K$ be two $S$-regular $R$-submodules of $S$. Then by (2), we have $[I K: I]=K$. If $I J \subseteq I K$, then $J \subseteq[I K: I]=K$.

$(3) \Rightarrow(1)$ This implication is obvious.

Proposition 2.4. Let $R \subseteq S$ be a ring extension, and let $I$ be a finitely generated $S$-regular ideal of $R$. Then $I$ is a cancellation ideal of $R \subseteq S$ if and only if $I$ is a quasi-cancellation ideal of $R \subseteq S$. 
Proof. Let $I$ be a finitely generated $S$-regular ideal of $R$. If $I$ is a cancellation ideal of the extension $R \subseteq S$, then obviously $I$ is an quasicancellation ideal of the extension $R \subseteq S$. Conversely, suppose that $I$ is a quasi-cancellation ideal of the extension $R \subseteq S$. Let $a_{1}, \ldots, a_{n} \in R$ be a set of generators of $I$. Let $B, C$ be two $S$-regular $R$-submodules of $S$ such that $I B \subseteq I C$. Let $b \in B$. Then $b I \subseteq I C$. So, for $1 \leqslant i \leqslant n$, we have $b a_{i}=\sum_{j=1}^{k} a_{j} c_{i j}$ with $c_{i j} \in C$ for $1 \leqslant j \leqslant k$. Furthermore, since $C S=S$, there exist $u_{1}, \ldots, u_{\ell} \in C$ and $s_{1}, \ldots, s_{\ell} \in S$ such that $u_{1} s_{1}+\cdots+u_{\ell} s_{\ell}=1$. Let $C^{\prime}$ be the $R$-submodule of $S$ generated by the elements of the set $\left\{u_{1}, \ldots, u_{n}, c_{i j}: 1 \leqslant i \leqslant n, 1 \leqslant j \leqslant k\right\}$. Let $B_{0}$ be the $R$-submodule of $S$ generated by $b$. Then $\left(B_{0}+\left(u_{1}, \ldots, u_{n}\right) R\right) I \subseteq I C^{\prime}$. It follows from the the equivalence $(1) \Leftrightarrow(3)$ of Proposition 2.3 that $B_{0}+\left(u_{1}, \ldots, u_{n}\right) R \subseteq C^{\prime}$ since $B_{0}+\left(u_{1}, \ldots, u_{n}\right)$ and $C^{\prime}$ are finitely generated $S$-regular ideal of $S$. Therefore, $b \in C^{\prime} \subseteq C$. Hence $B \subseteq C$ since $b$ was arbitrary chosen in $B$. This shows that $I$ is a cancellation ideal of the extension $R \subseteq S$.

Lemma 2.5. Let $R \subseteq S$ be a ring extension, and let $u_{1}, \ldots, u_{\ell} \in S$. Define the sets $E=\left(u_{1}, \ldots, u_{\ell}\right) R_{[\mathfrak{p}]}$ and $A=\left(u_{1}, \ldots, u_{\ell}\right) R$, where $\mathfrak{p}$ is a prime ideal of $R$. For any ideal $I$ of $R$, we have:

(1) $(A I)_{[\mathfrak{p}]}=(E I)_{[\mathfrak{p}]}$. In particular, $A_{[\mathfrak{p}]}=E_{[\mathfrak{p}]}$.

(2) $(E I)_{[\mathfrak{p}]}=\left(E I_{[\mathfrak{p}]}\right)_{[\mathfrak{p}]}$.

Proof. (1) First, observe that $A I \subseteq E I$. So $(A I)_{[\mathfrak{p}]} \subseteq(E I)_{[\mathfrak{p}]}$. Let $x \in$ $(E I)_{[\mathfrak{p}]}$. Then there exists $t \in R \backslash \mathfrak{p}$ such that $t x \in E I$. Therefore, $t x=$ $\sum_{i=1}^{n} e_{i} x_{i}$ for some $e_{i} \in E$ and $x_{i} \in I, 1 \leqslant i \leqslant n$. For each $1 \leqslant i \leqslant n$, write $e_{i}=\sum_{j=1}^{\ell} u_{j} y_{i j}$ with $y_{i j} \in R_{[\mathfrak{p}]}$ for $1 \leqslant j \leqslant \ell$. Let $s_{i j} \in R \backslash \mathfrak{p}$ such that $s_{i j} y_{i j} \in R, s_{i}=\prod_{j=1}^{\ell} s_{i j}$ and $s=\prod_{i=1}^{n}$. Then $s_{i} e_{i} \in A$. It follows that $(s t) x=\sum_{i=1}^{\ell}\left(s e_{i}\right) x_{i} \in A I$. Thus $x \in(A I)_{[\mathfrak{p}]}$ since $s t \in R \backslash \mathfrak{p}$. This shows that $(E I)_{[\mathfrak{p}]} \subseteq(A I)_{[\mathfrak{p}]}$. Hence $(A I)_{[\mathfrak{p}]}=(E I)_{[\mathfrak{p}]}$. In particular, if we take $I=R$, then we get $A_{[\mathfrak{p}]}=E_{[\mathfrak{p}]}$.

(2) The containment $(E I)_{[\mathfrak{p}]} \subseteq\left(E I_{[\mathfrak{p}]}\right)_{[\mathfrak{p}]}$ is clear since $E I \subseteq E I_{[\mathfrak{p}]}$. Let $x \in\left(E I_{[\mathfrak{p}]}\right)_{[\mathfrak{p}]}$. Then $t x \in E I_{[\mathfrak{p}]}$ for some $t \in R \backslash \mathfrak{p}$. Thus $t x=\sum_{i=1}^{k} v_{i} y_{i}$ with $v_{i} \in E$ and $y_{i} \in I_{[\mathfrak{p}]}$ for $1 \leqslant i \leqslant k$. Let $s_{i} \in R \backslash \mathfrak{p}$ such that $s_{i} y_{i} \in I$, and let $s=\prod_{i=1}^{k} s_{i}$. Then $(s t) x=\sum_{i=1}^{k} v_{i}\left(s y_{i}\right) \in E I$. It follows that $x \in(E I)_{[\mathfrak{p}]}$. Therefore, $(E I)_{[\mathfrak{p}]}=\left(E I_{[\mathfrak{p}]}\right)_{[\mathfrak{p}]}$

Theorem 2.6. Let $R \subseteq S$ be a ring extension, and let $I$ be a finitely generated $S$-regular ideal of $R$. The following statements are equivalent.

(1) $I$ is a quasi-cancellation ideal of the extension $R \subseteq S$. 
(2) For each prime ideal $\mathfrak{p}$ of $R$, and for each $S$-regular finitely generated $R_{[\mathfrak{p}]}$-submodule $E$ of $S$, we have $\left[(E I)_{[\mathfrak{p}]}: I_{[\mathfrak{p}]}\right]=E_{[\mathfrak{p}]}$.

Proof. (1) $\Rightarrow(2)$ Suppose that $I$ is a quasi-cancellation ideal of the extension $R \subseteq S$, and let $\mathfrak{p}$ be a prime ideal of $R$. Let $E$ be a finitely generated $S$-regular $R_{[\mathfrak{p}]}$-submodule of $S$. Then $E=\left(u_{1}, \ldots, u_{\ell}\right) R_{[\mathfrak{p}]}$ for some elements $u_{1}, \ldots, u_{\ell}$ of $S$. Let $A$ be the $R$-submodule of $S$ generated by $u_{1}, \ldots, u_{\ell}$. Then by Proposition 2.3 and Proposition 2.4 , we have $[A I: I]=A$. It follows from $[6$, Proposition $2.1(4)]$ that $\left[(A I)_{\mathfrak{p}}: I_{[\mathfrak{p}]}\right]=A_{[\mathfrak{p}]}$. Hence by Lemma 2.5 , we have $\left[\left(E I_{[\mathfrak{p}]}\right)_{[\mathfrak{p}]}: I_{[\mathfrak{p}]}\right]=\left[(E I)_{\mathfrak{p}}: I_{[\mathfrak{p}]}\right]=\left[(A I)_{\mathfrak{p}}\right.$ : $\left.I_{[\mathfrak{p}]}\right]=A_{[\mathfrak{p}]}=E_{[\mathfrak{p}]}$.

$(2) \Rightarrow(1)$ Suppose that the statement (2) is true. Let $A$ be an $S$-regular finitely generated $R$-submodule of $S$, and let $\mathfrak{p}$ be a prime ideal of $R$. Let $E=A R_{[\mathfrak{p}]}$. Then by Lemma 2.5, we have $(A I)_{[\mathfrak{p}]}=(E I)_{[\mathfrak{p}]}$ and $A_{[\mathfrak{p}]}=E_{[\mathfrak{p}]}$. So, by hypothesis we have $A_{[\mathfrak{p}]}=E_{[\mathfrak{p}]}=\left[(E I)_{[\mathfrak{p}]}: I_{[\mathfrak{p}]}\right]=\left[(A I)_{[\mathfrak{p}]}: I_{[\mathfrak{p}]}\right]$. But by $[6$, Proposition $2.1(4)]$, we have $\left[(A I)_{[\mathfrak{p}]}: I_{[\mathfrak{p}]}\right]=[(A I): I]_{[\mathfrak{p}]}$. Therefore, $A_{[\mathfrak{p}]}=[(A I): I]_{[\mathfrak{p}]}$ for each prime ideal $\mathfrak{p}$ of $R$. It follows from Remark 1.1 that $[A I: I]=A$. Therefore, by the equivalence $(1) \Leftrightarrow(2)$ of Proposition 2.3, $I$ is a quasi-cancellation ideal of the extension $R \subseteq S$.

In their book [5], Knebusch and Zhang defined the notion of Prüfer extension using valuation ring [5, Definition 1, p. 46]. Several characterizations of a Prüfer extension are given in [5, Theorem 5.2, p. 47]. For the purpose of this work, we will use the following: a ring extension $R \subseteq S$ is called Prüfer extension if $R$ is integrally closed in $S$ and $R[\alpha]=R\left[\alpha^{n}\right]$ for any $\alpha \in S$ and any $n \in \mathbb{N}$.

Lemma 2.7. [5, Theorem 1.13, p. 91] If a ring extension $R \subseteq S$ is a Prüfer extension, then every finitely generated $S$-regular $R$-submodule of $S$ is $S$-invertible.

Proposition 2.8. Let $R \subseteq S$ be a ring extension, and let $I$ be an $S$-regular ideal of $R$.

(1) If $I$ is a cancellation ideal of the extension $R \subseteq S$, then $[I: I]=R$.

(2) If the extension $R \subseteq S$ is Prüfer, then the converse of statement (1) is also true (i.e. in a Prüfer extension $R \subseteq S$, if $I$ is an $S$-regular ideal satisfying $[I: I]=R$, then $I$ is a quasi-cancellation ideal).

Proof. (1) The proof follows directly from the equivalence (1) $\Leftrightarrow$ (2) of Theorem 2.3. It suffices to take $J=R$.

(2) Suppose that the extension $R \subseteq S$ is Prüfer, and let $I$ be an $S$-regular ideal of $R$ such that $[I: I]=R$. Let $A$ be an $S$-regular finitely 
generated $R$-submodule of $S$. Then by Lemma 2.7, $A$ is $S$-invertible. We show that $A[I: I]=[A I: I]$. Let $x \in[A I: I]$. Then $x I \subseteq A I$. Hence $x I A^{-1} \subseteq I$. Thus $x A^{-1} \subseteq[I: I]$. It follows that $x \in A[I: I]$. On the other hand, let $y=\sum_{i=1}^{k} a_{i} v_{i} \in A[I: I]$ with $a_{i} \in A$ and $v_{i} \in[I: I]$ for $1 \leqslant i \leqslant k$. Then $v_{i} I \subseteq I$. Hence $a_{i} v_{i} I \subseteq A I$. Therefore, $a_{i} v_{i} \in[A I: I]$. So $y=\sum_{i=1}^{k} a_{i} v_{i} \in[A I: I]$. This shows that $[A I: I]=A[I: I]$. Hence $[A I: I]=A[I: I]=A R=A$. Hence, by the equivalence $(1) \Leftrightarrow(2)$ of Proposition 2.3, $I$ is a quasi-cancellation ideal of the extension $R \subseteq S$.

Let $R \subseteq S$ be a ring extension. A nonzero $S$-regular ideal $I$ of $R$ is called $m$-canonical ideal of the extension $R \subseteq S$ if $[I:[I: J]]=J$ for all $S$-regular ideal $J$ of $R$. Properties of m-canonical ideals of a ring extension are studied in [7].

Corollary 2.9. Any m-canonical ideal of a Prüfer extension is a quasicancellation ideal.

Proof. If $I$ is an $m$-canonical ideal of a Prüfer extension $R \subseteq S$, then by [7, Proposition 2.3], we have $[I: I]=R$. It follows from Proposition 2.8(2) that $I$ is a quasi-cancellation ideal of the extension $R \subseteq S$.

Lemma 2.10. Let $R \subseteq S$ be a ring extension, and let $I$ be an $S$-regular ideal of $R$ which is a cancellation ideal of $R \subseteq S$. If $I=(x, y)+A$, where $A$ is an ideal of $R$ containing $\mathfrak{m} I$ for some maximal ideal $\mathfrak{m}$ of $R$, then $I=(x)+A$ or $I=(y)+A$.

Proof. Let $J=\left(x^{2}+y^{2}, x y, x A, y A, A^{2}\right) R$. Then $I J=I^{3}$. Observe that $I^{2}$ is $S$-regular since $I^{2} S=I(I S)=I S=S$. Also, from the equality $I J=I^{3}$ we have $(I J) S=I^{3} S=I(I S)=I S=S$. So $J S=S$. This shows that $J$ is an $S$-regular ideal of $R$. It follows from the equation $I J=I^{3}$ and the fact that $I$ is a cancellation ideal of the extension $R \subseteq S$ that $J=I^{2}$. Thus $x^{2}=t\left(x^{2}+y^{2}\right)+$ terms from $\left(x y, x A, y A, A^{2}\right)$, with $t \in R$. Suppose that $t \in \mathfrak{m}$. Then $x^{2} \in\left(y^{2}, x y, x A, y A, A^{2}\right)$, since $t x \in \mathfrak{m} I \in A$. Let $K=(y)+A$. Then $I^{2}=I K$. Furthermore, from the equality $I K=I^{2}$, we have $K(I S)=I^{2} S$. Hence $K S=S$. Therefore, $K$ is an $S$-regular ideal of $S$. It follows that $I=K$ since $I$ is a cancellation ideal of the extension $R \subseteq S$. The rest of the proof is similar to the proof of [2, Lemma].

Proposition 2.11. Let $R \subseteq S$ be a ring extension, and let $I$ be a nonzero $S$-regular ideal of $R$. If I is a cancellation ideal of the extension $R \subseteq S$, then for each maximal ideal $\mathfrak{m}$ of $R$, there exists a $\in R$ such that $I_{[\mathfrak{m}]}=(a)_{[\mathfrak{m}]}$. 
Proof. Suppose that $I$ is a cancellation ideal of the ring extension $R \subseteq S$, and let $\mathfrak{m}$ be a maximal ideal of $R$. Suppose that $I \subseteq \mathfrak{m}$. Then by Lemma 2.10, and the proof of [2, Theorem], there exists $a \in I$ such that for each $b \in I,(1-u) b=r a$ for some $u \in \mathfrak{m}$ and $r \in R$. Therefore, $(1-u) b \in(a)$. So $b \in(a)_{[\mathfrak{m}]}$. This shows that $I_{[\mathfrak{m}]} \in(a)_{[\mathfrak{m}]}$. On the other hand, the equality $(a)_{[\mathfrak{m}]} \subseteq I_{[\mathfrak{m}]}$ is always true. Thus $I_{[\mathfrak{m}]}=(a)_{[\mathfrak{m}]}$. If $I \nsubseteq \mathfrak{m}$, then $I_{[\mathfrak{m}]}=(1)_{[\mathfrak{m}]}=R_{[\mathfrak{m}]}$. In fact, for $x \in R_{[\mathfrak{m}]}$, there exists $s \in R \backslash \mathfrak{m}$ such that $s x \in R$. Thus $(s t) x \in I$ for each $t \in I \backslash \mathfrak{m}$. It follows that $x \in I_{[\mathfrak{m}]}$.

Theorem 2.12. Let $R \subseteq S$ be a ring extension, and let $I$ be a nonzero finitely generated $S$-regular ideal of $R$. The following statements are equivalent.

(1) $I$ is a cancellation ideal of the extension $R \subseteq S$.

(2) $I$ is a quasi-cancellation ideal of the extension $R \subseteq S$.

(3) $I$ is an $S$-invertible ideal of $R$.

(4) $I R[X]$ is a cancellation ideal of the extension $R[X] \subseteq S[X]$.

Proof. The equivalence (1) $\Leftrightarrow(2)$ is the result of Theorem 2.4.

$(1) \Rightarrow(3)$ Suppose that $I$ is a cancellation ideal of the extension $R \subseteq S$, and let $\mathfrak{m}$ be a maximal ideal of $R$. By the previous proposition, $I_{[\mathfrak{m}]}=(a)_{[\mathfrak{m}]}$ for some $a \in R$. It follows that $\left(I_{[\mathfrak{m}]}\right)_{\mathfrak{m}_{[\mathfrak{m}]}}=\left((a)_{[\mathfrak{m}]}\right)_{\mathfrak{m}_{[\mathfrak{m}]}}$. But by $\left[5\right.$, Lemma $2.9\left(\right.$ b), p. 28], we have $I_{\mathfrak{m}}=\left(I_{[\mathfrak{m}]}\right)_{\mathfrak{m}_{[\mathfrak{m}]}}$ and $(a)_{\mathfrak{m}}=\left((a)_{[\mathfrak{m}]}\right)_{\mathfrak{m}_{[\mathfrak{m}]}}$. Hence $I_{\mathfrak{m}}=(a)_{\mathfrak{m}}$. This shows that $I$ is locally principal. It follows from [5, Proposition 2.3, p. 97] that $I$ is $S$-invertible.

$(3) \Rightarrow(1)$ This implication is obvious.

$(3) \Rightarrow(4)$ Suppose that $I$ is an $S$-invertible ideal of the extension $R \subseteq S$. First, note that $(I R[X])(S[X])=S[X]$ since $I S=S$. Hence $I R[X]$ is an $S[X]$-regular ideal of $R[X]$. Let $J$ be the $R$-submodule of $S$ such that $I J=R$. Then $(I R[X])(J R[X])=R[X]$. This shows that $I R[X]$ is an $S[X]$-invertible ideal of $R[X]$. It follows from the equivalence $(1) \Leftrightarrow(3)$ that $I R[X]$ is a cancellation ideal of the extension $R[X] \subseteq S[X]$.

$(4) \Rightarrow(1)$ Suppose that $I R[X]$ is a cancellation ideal of the extension $R[X] \subseteq S[X]$. Let $J$ be an $S$-regular ideal of $R$. Then by the equivalence $(1) \Leftrightarrow(2)$ of Proposition 2.3, we have $[(I R[X])(J R[X]): I R[X]]=J R[X]$.

We show that $[I J: I]=J$. First, note that the containment $J \subseteq[I J$ : $I]$ is always true. Let $u \in[I J: I]$. Then $u I \subseteq I J$. Therefore, $u I R[X] \subseteq$ $(I J) R[X] \subseteq(I R[X])(J R[X])$. Hence $u \in[(I R[X])(J R[X]): I R[X]]=$ $J R[X]$. It follows that $u \in J R[X] \cap S=J$. This shows that $[I J: I] \subseteq$ $J$. Hence $[I J: I]=J$. It follows from the equivalence $(1) \Leftrightarrow(2)$ of Proposition 2.3 that $I$ is a cancellation ideal of the extension $R \subseteq S$. 
Corollary 2.13. Let $R \subseteq S$ be a ring extension, and let $I$ be a finitely generated $S$-regular ideal of $R$. If $I$ is a cancellation ideal of the extension $R \subseteq S$, then $I_{[\mathfrak{m}]}$ is a cancellation ideal of the extension $R_{[\mathfrak{m}]} \subseteq S$ for each maximal ideal $\mathfrak{m}$ of $R$.

Proof. Let $I$ be a finitely generated $S$-regular ideal of $R$, and let $\mathfrak{m}$ be a maximal ideal of $R$. Suppose that $I$ is a cancellation ideal of the extension $R \subseteq S$. Then by the previous theorem, $I$ is $S$-invertible. Let $J$ be an $R$-submodule of $S$ such that $I J=R$. Then $I_{[\mathfrak{m}]} J_{[\mathfrak{m}]} \subseteq(I J)_{[\mathfrak{m}]} \subseteq R_{[\mathfrak{m}]}$. Furthermore, since $I S=S$, there exist $x_{i} \in I$ and $y_{i} \in J, 1 \leqslant i \leqslant \ell$, such that $1=\sum_{i=1}^{\ell} x_{i} y_{i}$. Let $u \in R_{[\mathfrak{m}]}$. There exists $t \in R \backslash \mathfrak{m}$ such that $t u \in R$ and $u=\sum_{i=1}^{\ell}\left(u x_{i}\right) y_{i}$. But for $1 \leqslant i \leqslant \ell, t\left(u x_{i}\right)=(t u) x_{i} \in I$ since $t u \in R$ and $x_{i} \in I$. It follows that $u x_{i} \in I_{[\mathfrak{m}]}$. Therefore, $u=\sum_{i=1}^{\ell}\left(u x_{i}\right) y_{i} \in$ $I_{[\mathfrak{m}]} J \subseteq I_{[\mathfrak{m}]} J_{[\mathfrak{m}]}$. This shows that $R_{[\mathfrak{m}]} \subseteq I_{[\mathfrak{m}]} J_{[\mathfrak{m}]}$. Thus $I_{[\mathfrak{m}]} J_{[\mathfrak{m}]}=R_{[\mathfrak{m}]}$. Hence $I_{[\mathfrak{m}]}$ is an $S$-invertible $R_{[\mathfrak{m}]}$-submodule of $S$. It follows that $I_{[\mathfrak{m}]}$ is a cancellation ideal of the extension $R_{[\mathfrak{m}]} \subseteq S$, since an invertible ideal of ring extension is always a cancellation ideal.

\section{References}

[1] Anderson, D.D., Anderson, D. F. (1984). Some remarks on cancellation ideals. Math Japonica. 29 (6), pp 879-886.

[2] Anderson, D.D., Roitman, M. (1997). A characterization of cancellation ideals. Proc. Amer. Math. Soc. No. 10, pp 2853 - 2854.

[3] Fuchs, L.; Salce, L. (2001) Modules over non-Noetherian domains. Mathematical Surveys and Monographs, Vol. 84. American Mathematical Society, Providence, RI. $\mathrm{xvi}+613 \mathrm{pp}$.

[4] Gabelli, S; Houston, E. (2000). Ideal theory in pullbacks. In Chapman S. T; Glaz S., eds. Non-Noetherian commutative ring theory. Math. Appl., Vol 520. Dordrecht: Kluwer Acad. Publ., pp. 199-227.

[5] Knebusch, M., Zhang, D. (2002). Manis valuations and Prüfer extensions I. Lecture Notes in Mathematics, Vol. 1791. Berlin: Springer-Verlag.

[6] Paudel, L., Tchamna, S. (2018). On the saturation of submodules. Algebra and Discrete Mathematics, Vol. 26, No. 1, pp. 110 - 123

[7] Tchamna, S. (2017). Multiplicative canonical ideals of ring extensions. Journal of Algebra and Its Appl. Vol. 16, No. 4:170069.

\section{CONTACT INFORMATION}

\section{Simplice Tchamna Department of Mathematics}

Georgia College, Milledgeville, GA, USA

E-Mail(s): simplice.tchamna@gcsu.edu

Received by the editors: 26.07.2019

and in final form 30.10.2020. 